\title{
Non-linear field generated by a distribution of conductors in a spiral FFAG
}

\author{
Bruno Autin CERN, Emmanuel Froidefond LPSC/IN2P3/CNRS,
}

\begin{abstract}
FFAGs accelerators re-appeared fifteen years ago to be of interest for new accelerator projects. These synchrotrons allow fast acceleration because of the fixed field magnets. To compensate the beam trajectory radius increase which would happen, the radial gradient follows a non-linear law $B_{0}\left(r / R_{0}\right)^{k}$. Amongst several methods, in the frame of the RACCAM project in France, a scaling spiral FFAG ring is developed for medical applications. Magnets have spiral edges to make beam focusing, and here is described the design study for flat horizontal poles that allow a low variation of the vertical tune. This study presents how to produce $B_{0}\left(r / R_{0}\right)^{k}$ with distributed polar conductors.
\end{abstract}

Index Terms-FFAG synchrotron, spiral magnet, polar conductor distribution, non-linear field gradient

\section{INTRODUCTION}

$\mathrm{F}$ FAGs have been invented during the 1950s at MURA (this lab was dismantled in the late 1960s). Three electron models were constructed, including a spiral one, up to $50 \mathrm{MeV}$ in 1961. During the late 1990s, FFAG synchrotron accelerators have come to new interest for accelerator projects in the fields of neutrino factory for fast acceleration, proton beams and more recently of medical accelerators. In these accelerators the dipole field remains constant with time, and to compensate the increasing beam trajectory radius the magnetic field follows a radial law such as $\mathrm{B}_{0}\left(\mathrm{r} / \mathrm{R}_{0}\right)^{\mathrm{k}}$. To match this law, two main methods were imagined : a pole shaped yoke and distributed conductors on flat poles. The main advantage of this second technique is to maintain a low variation of the vertical tune,which varies with the rate radius/gap height. The ideal situation would be to increase gap height with radius, so that the vertical tune remains constant during the acceleration.

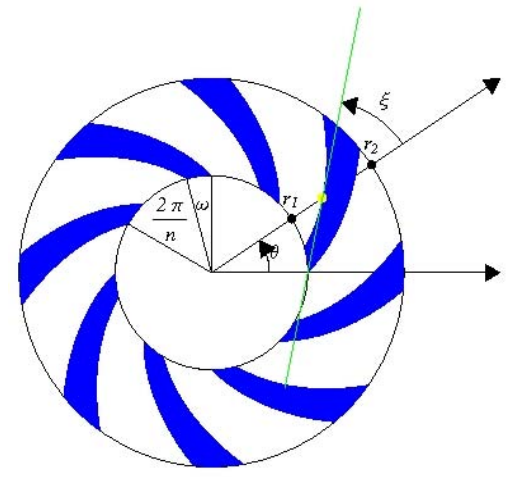

Fig. 1. Schematic diagram of an 8 cells FFAG synchrotron ring..

In the frame of the RACCAM project in France, a scaling spiral FFAG synchrotron ring is developed for medical purpose. Injection energy is $17 \mathrm{MeV}$ and ejection energy is $180 \mathrm{MeV}$ with 8 cells (a 10 cells design is still considered for practical and beam dynamics reasons). In scaling FFAGs, beam focusing is usually made using alternated dipole field gradient as in recent Japanese demonstrators [1]. Here it is made with spiral edges of magnets, which allows a more compact machine. Due to spiral edges, the radial field law is multiplied by a function where the spiral angle $\xi$ appears :

$$
B=B_{0}\left(\frac{r}{R_{0}}\right)^{k} F\left(\theta-\tan \xi \ln \left(\frac{r}{R_{0}}\right)\right)
$$

$\theta$ is the polar coordinates angle. The function $\mathrm{F}$ has no meaning in a $2 \mathrm{~d}$ calculation.

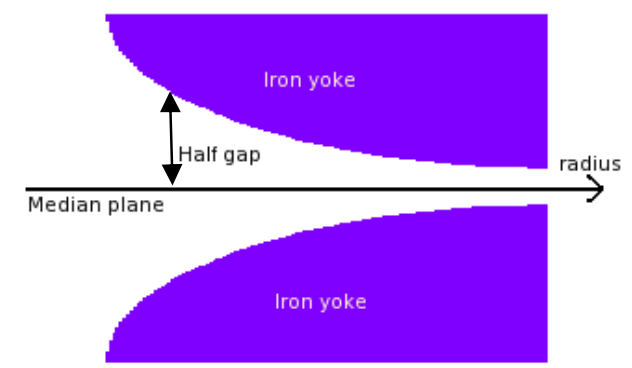

Fig. 2. Pole shaped iron yoke that produces a field law $\mathrm{B}_{0}\left(\mathrm{r} / \mathrm{R}_{0}\right)^{\mathrm{k}}$ with fixed $\mathrm{k}$. That prevents from small adjustments to tune the accelerator. Gap height diminishes with radius, and hence with energy.

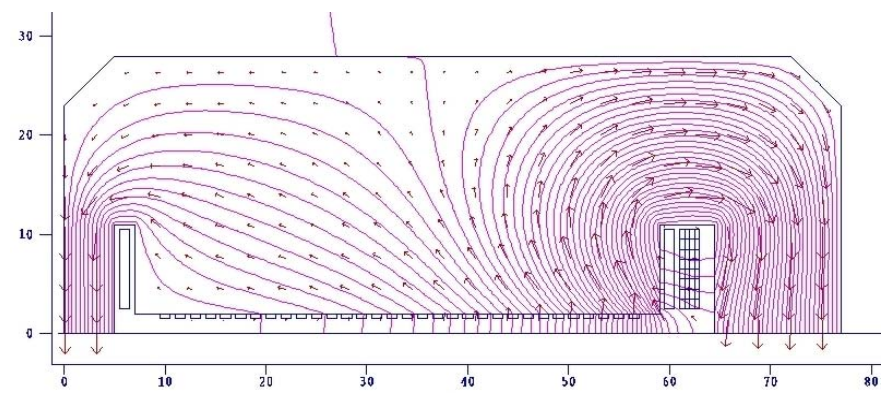

Fig. 3. Flat pole design with distributed conductors. Flux in the iron yoke is concentrated in the large radius area. With this solution, it is possible to build a system with variable intensity in the conductors, which would allow adjustable $\mathrm{k}$ and maybe facilitate a variable extraction energy.

\section{FROM THE FIELD TO CURRENT INTENSITIES}

Previously to any calculation, the first idea that comes to mind to calculate current intensities should be forgotten : the current law does not follow the field law. If the current law really has an exponential shape, the exponent to $r$ is different from k. From Biot and Savart law, one can write the field components in the median plane for a simple straight wire as follow : 
- horizontal $: B_{x}=\frac{\mu_{0} I}{2 \pi} \frac{\eta}{(\xi-x)^{2}+\eta^{2}}$

- vertical : $B_{y}=\frac{\mu_{0} I}{2 \pi} \frac{\xi-x}{(\xi-x)^{2}+\eta^{2}}$.

$(\xi, \eta)$ are the wire coordinates, and $(\mathrm{x}, \mathrm{y})$ the coordinates of the observation point (see fig.3).

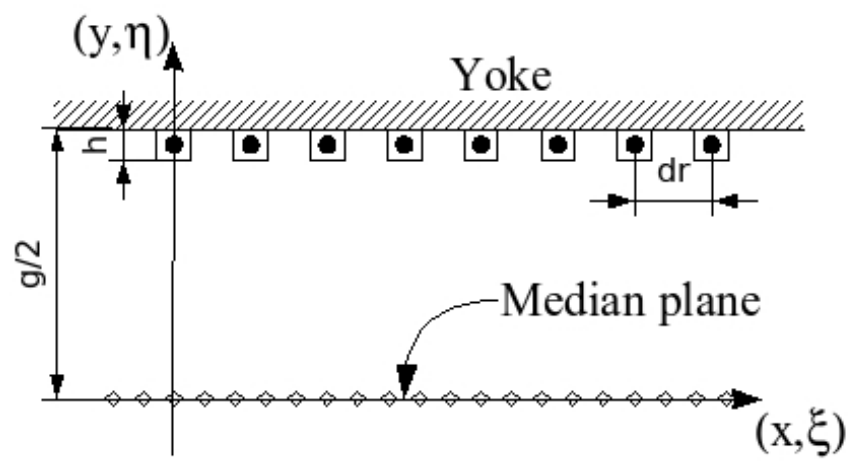

Fig. 3. Schematic diagram of the $2 \mathrm{~d}$ geometry. The iron yoke is not included in the calculation process even if it is represented.

If $\mathrm{N}$ is the number of conductors and $\mathrm{P}$ the number of observation points, then the field at any of the $\mathrm{P}$ points is the sum of the contribution of each wire. This constitutes a system of linear equation, which can be written as a simple linear matrix equation : $\boldsymbol{A} . \boldsymbol{I}-\boldsymbol{B}=\mathbf{0}$.

Each matrix $A$ of this equation is written for either the horizontal or the vertical components as follow :

$$
\begin{aligned}
& A_{x}=\frac{\mu_{0}}{2 \pi}\left(\begin{array}{ccc}
\frac{\eta_{1}}{\left(\xi_{1}-x_{1}\right)^{2}+\eta_{1}{ }^{2}} & \cdots & \frac{\eta_{N}}{\left(\xi_{N}-x_{1}\right)^{2}+\eta_{N}{ }^{2}} \\
\vdots & \ddots & \vdots \\
\frac{\eta_{1}}{\left(\xi-x_{P 1}\right)^{2}+\eta_{1}{ }^{2}} & \cdots & \frac{\eta_{N}}{\left(\xi_{N}-x_{P}\right)^{2}+\eta_{N}{ }^{2}}
\end{array}\right) \\
& A_{y}=\frac{\mu_{0}}{2 \pi}\left(\begin{array}{ccc}
\frac{\xi_{1}-x_{1}}{\left(\xi_{1}-x_{1}\right)^{2}+\eta_{1}^{2}} & \cdots & \frac{\xi_{N}-x_{1}}{\left(\xi_{N}-x_{1}\right)^{2}+\eta_{N}^{2}} \\
\vdots & \ddots & \vdots \\
\frac{\xi_{1}-x_{P}}{\left(\xi_{1}-x_{P}\right)^{2}+\eta_{1}^{2}} & \cdots & \frac{\xi_{N}-x_{P}}{\left(\xi_{N}-x_{P}\right)^{2}+\eta_{N}^{2}}
\end{array}\right)
\end{aligned}
$$

This system is reversible but over-determined if $\mathrm{P}>\mathrm{N}$, which means the system has more than one unique solution or no solution. Due to the dimensions of the magnet gap, which height's is much smaller than its radial extent, $A_{x}$ and $A_{y}$ are ill-conditioned in most cases. This second point is another reason why the difficulty to solve the system is increased. When $\mathrm{P}>\mathrm{N}$, the matrix equation is multiplied by the transpose of $\mathrm{A}$ to get a squared matrix, and $\mathrm{A}^{\mathrm{T}} \mathrm{A}$ is more ill-conditioned than $\mathrm{A}$.

\section{2D CALCULATION}

\section{A. Solving the equations system}

In a $2 \mathrm{~d}$ calculation, because it can not be determined :

$$
F\left(\theta-\tan \xi \ln \left(\frac{r}{R_{0}}\right)\right)=1 \text {. }
$$

A Mathematica function was written to produce matrix A for several situations, especially it can consider the width and thickness of conductors. Then field components change as follow :
- Horizontal : $\boldsymbol{B}_{x}=\frac{\mu_{0} I}{2 \pi} \int_{h(\xi)}^{g / 2} \int_{p}^{q} \frac{\eta}{(\xi-x)^{2}+\eta^{2}} d \eta d \xi$

$$
=\int_{p}^{q}\left(-\arctan \left[\frac{g / 2}{\xi-x}\right]+\arctan \left[\frac{h[\xi !}{\xi-x}\right]\right) d \xi
$$

- vertical. : $B_{y}=\frac{\mu_{0} I}{2 \pi} \int_{h(\xi)}^{g / 2} \int_{p}^{q} \frac{\xi-x}{(\xi-x)^{2}+\eta^{2}} d \eta d \xi$

$$
\cdots \cdots=\frac{1}{2} \int_{p}^{q}\left(\ln \left[g^{2}+(\xi-x)^{2}\right]-\ln \left[(\xi-x)^{2}+h[\xi]\right]\right) d \xi
$$

$\mathrm{g}$ is the gap height (see fig.3), and $\mathrm{h}(\xi$,) is the height under conductors that allow varying the height of conductors. This is when useful current intensity reaches a high value, because that allows increasing the size of the conductor in order to diminish the current density. $\mathrm{p}$ and $\mathrm{q}$ are the limits of the conductor's width.

The equations system keeps the same shape when these integrals are included in the calculation. To solve this linear system, least square methods do not work because the matrix condition number is greater than 1000 in most cases. The only method which allows finding a solution is using norms minimization. The one giving best results is the one-norm minimization, which means :

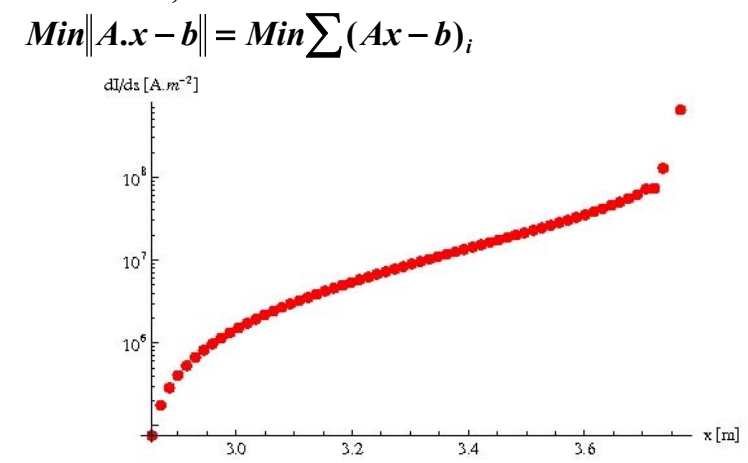

Fig. 4. Current density calculation results considering polar conductors only.

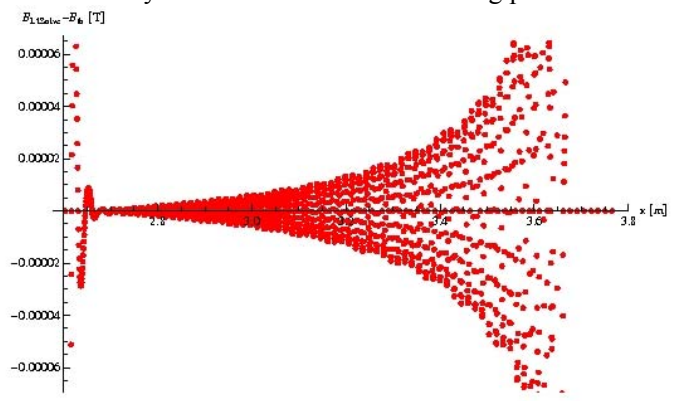

Fig. 5. Difference between the theoretical field and the one determined with the Mathematica function.

For a field law with $\mathrm{B}_{\max }=1.5 \mathrm{~T}$, most current densities remains under $100 \mathrm{MA} . \mathrm{m}^{-2}$, which is still a high value but cooling is achievable. At high currents, the field precision is still lower than $10^{-4}$.

\section{B. POISSON simulation}

Only polar distributed conductors are considered with geometry parameters found in table I. A transverse cut schematic diagram of the model is shown in figure 3. Minimum and maximum field intensities correspond to what was expected, but oscillations, which can be observed more precisely looking at $\mathrm{db}_{\mathrm{y}} / \mathrm{dx}$ (fig. 6), appear clearly. Despite this, a particle beam simulation has shown relative effects on dynamic tunes lower than $10^{-3}$. 


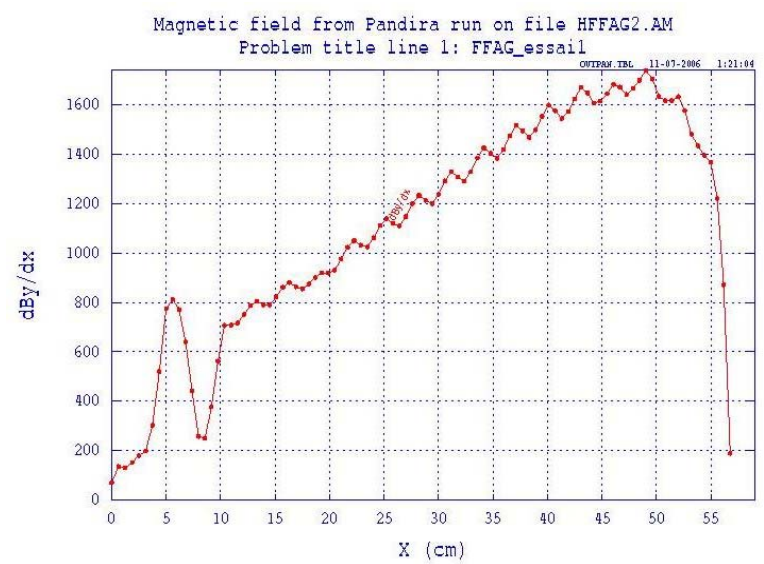

Fig. 6. Graphs results of a $2 \mathrm{~d}$ finite elements POISSON simulation. Field is measured in Gauss.

The amplitude of oscillation diminishes when the distance between conductors is reduced to zero.

\section{3D CALCULATION}

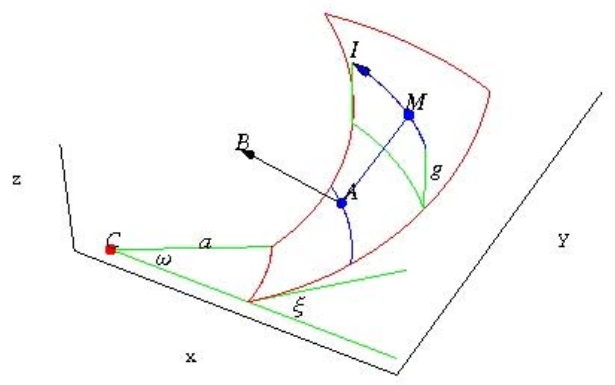

Conductors infinitely thin and located at height $\mathrm{g} / 2$ have finite length. Both observation points and conductors lie on arcs circles. For a magnet with a spiral angle $\xi$ and a minimum radius a, extremities of an arc at opening angle $\omega$ are located at angles:

- $\theta_{1}=\ln \left(\frac{r}{a}\right) \tan \xi$

- $\theta_{2}=\omega+\ln \left(\frac{r}{a}\right) \tan \xi$

With this configuration, the field expression must be rewritten. The elementary conductor, of length $\mathrm{dl}$ at point $\mathrm{M}$ with a current intensity I, creates at point $\mathrm{A}$ a field $\mathrm{dB}$ given by the Biot and Savart law :

$$
d B=\frac{\mu_{0} I}{4 \pi} \frac{d l \wedge u}{d^{2}}
$$

where $d=\sqrt{r^{2}+R^{2}+\frac{g^{2}}{4}-2 r R \cos (\alpha-\theta)}$.

By adding contributions of conductors located at symmetrically at equal distance from the median plane, the field becomes purely vertical. The field is then :

$$
\begin{aligned}
\boldsymbol{B}_{z} & =\frac{\mu_{0} I R}{\pi} \int_{\theta_{1}}^{\theta_{2}} \frac{R-r \cos (\alpha-\theta)}{\left(r^{2}+R^{2}+g^{2} / 4-2 r R \cos (\alpha-\theta)\right)^{3 / 2}} d \theta \\
& =\frac{\mu_{0} I}{\pi r}\left(\boldsymbol{F}\left(\theta_{2}\right)-F\left(\theta_{1}\right)\right)
\end{aligned}
$$

where $\mathrm{F}(\theta)$ is a continuous function. Figure 7 show the field of the arc conductor at different radii. Clearly, the field maximum does not follow the median spiral, and reaches regions out of the limits of the spiral edges.

\section{A. Loop conductors}

A loop conductor consists in two arc conductor of same radius separated by a distance $\mathrm{g}$, one with intensity $+\mathrm{I}$, and other one with $-\mathrm{I}$. The distance between loops is $\mathrm{g} / \mathrm{n}$ so that two adjacent loops overlap.

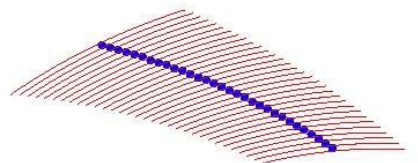

Fig. 7. Schematic diagram of loop conductors. Blue dots are observation points distributed on the median spiral.
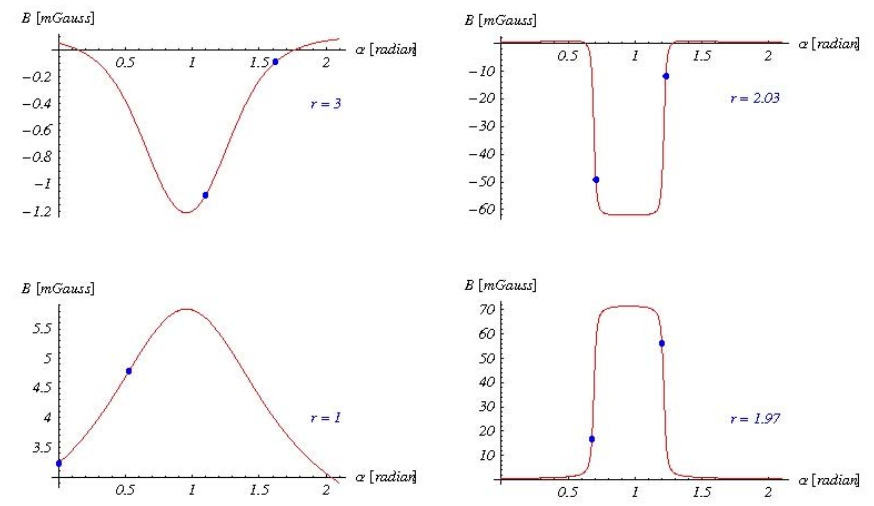

Fig. 8. Graphs of the field produced by an arc conductor at different radii. Blue dots indicate the spiral edges.

Observation points are located where the field due to a loop has its maximum. The matrix $\mathrm{A}$ is then well conditioned.

\section{B. Calculation of current intensities}

Current intensities carried out with loop conductors methods reach very high values, up to 1MA. The precision of the calculation increases while reducing the distance between adjacent loops. But there are still small oscillations which can be eliminated by anticipating field errors with a polynomial function $\mathrm{f}$. $\mathrm{f}$ reduces the field law as follow : $\mathrm{B}_{0}\left(\mathrm{r} / \mathrm{R}_{0}\right)^{\mathrm{k}}(1-\mathrm{f})$. The error is then limited to one per mil at maximum (see fig. 9).

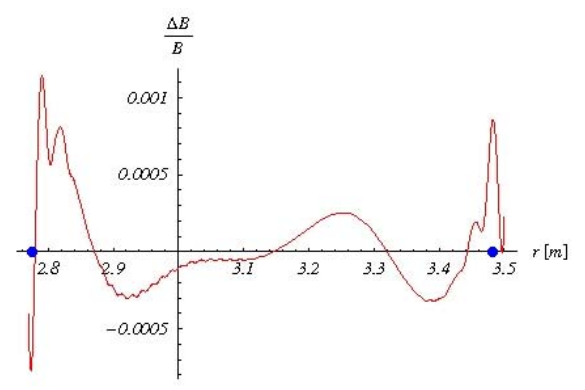

Fig. 9. Graphs of the field produced by a single conductor (blue) and a loop (red).

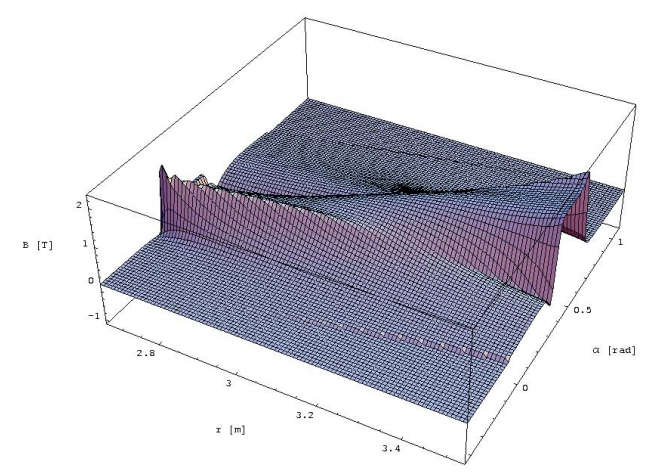

Fig. 10. $3 \mathrm{~d}$ map of the field calculated using loop conductors. 


\section{3D FINITE ELEMENT MODELS}

The $3 \mathrm{~d}$ finite element model is made on the basis of an 8 cells spiral FFAG ring from RACCAM project, using the BH curve of "good quality magnet steel" proposed in OPERA3d.

TABLE I 8 CELLS FFAG PARAMETERS

\begin{tabular}{ll}
\hline \hline Particle & Proton \\
\hline Number of cells & 8 \\
\hline Injection energy & $17 \mathrm{MeV}$ \\
\hline Ejection energy & $180 \mathrm{MeV}$ \\
\hline Injection radius & $3.4825 \mathrm{~m}$ \\
\hline Ejection radius & $2.7759 \mathrm{~m}$ \\
\hline $\mathrm{k}$ & 4.415 \\
\hline Field at ejection & $1.5 \mathrm{~T}$ \\
\hline Opening angle $\omega$ & $17.1^{\circ}$ \\
\hline Spiral angle $\xi$ & $50.36^{\circ}$ \\
\hline Gap height $\mathrm{g}$ & $0.03 \mathrm{~m}$ \\
\hline \hline
\end{tabular}

\section{A. Polar conductors only}

The basic orientation of arcs, taken from the pole shaping method, makes the centre of the machine ring the center of all arcs. But the finite elements simulation shows a large concentration of the flux in the closed angle area of the pole surface, where the iron yoke is saturated. This comes from the problem described in the previous section : a single wire produces a magnetic field perpendicularly to its axis.

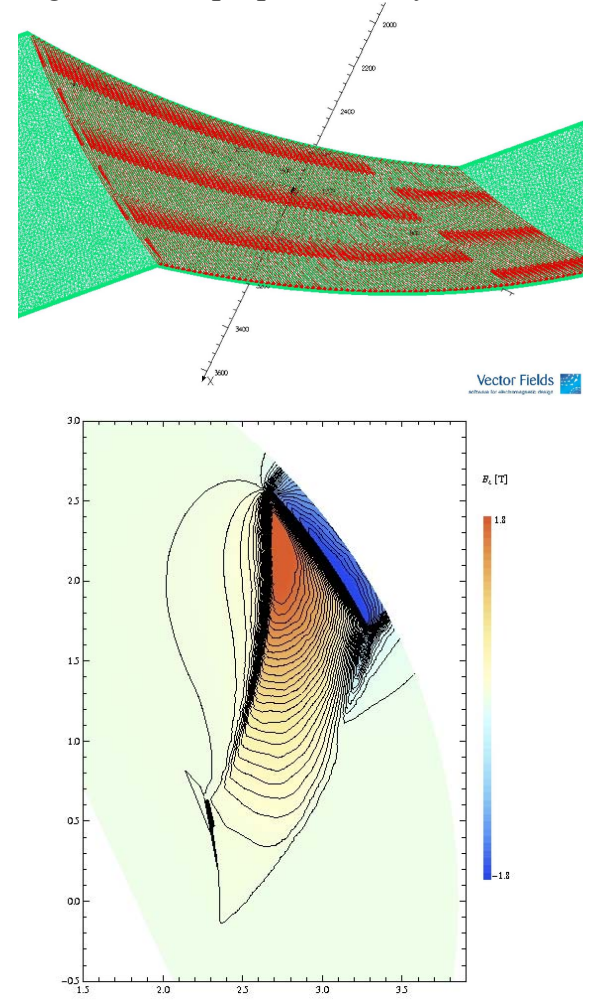

Fig. 11. OPERA3d view of distributed conductor and the yoke (green), and the field map of the vertical component in the median plane. The field is generated by distributed conductors only.

Rotating arcs, in order to make their median tangential to the spiral axis, makes the flux then totally concentrated in the gap, and the iron yoke is no more saturated. But this geometry does not allow keeping a constant field on a circular beam trajectory, which is mandatory during the acceleration process.

\section{B. Polar conductors and a main coil}

The main reason why the flux concentrates in a small region is the concentration of conductors with large current intensities in this area. In order to minimize these intensities, the distributed conductors can be added a main coil which produces a uniform field. The field to match with distributed conductors is then :

$$
B=B_{0}\left(\frac{r}{R_{0}}\right)^{k} F\left(\theta-\tan \xi \ln \left(\frac{r}{R_{0}}\right)\right)-B_{1},
$$

where $\mathrm{B}_{1}=1 / 2\left(\mathrm{~B}_{\max }+\mathrm{B}_{\min }\right)$.

\section{Using $3 d$ calculation results}

Even if the current densities predicted with the $3 d$ calculation are out of reasonable limits, it is interesting to look at the results that it gives in a finite element model. Machine parameters are still those of table I.

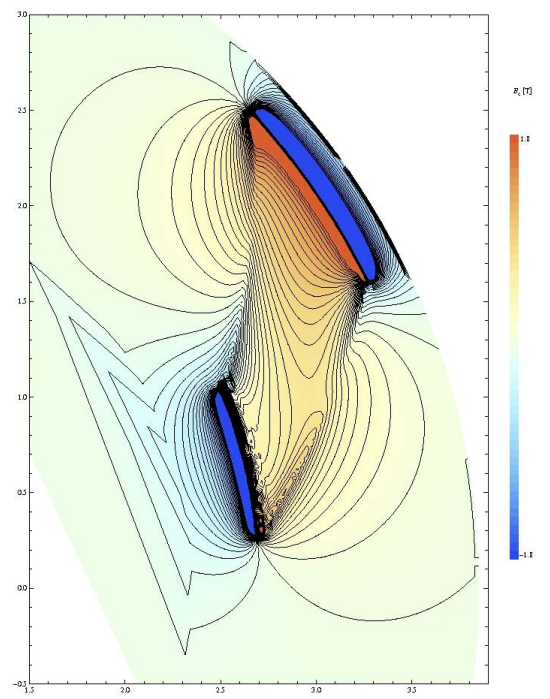

Fig. 12. Graphs results of a $2 \mathrm{~d}$ finite elements POISSON simulation. Field is measured in Gauss (straight lines at bottom left are due to failing display).

Comparing the field map in figure 12 to the one in figure10, agreement clearly appears on the field shape. Amplitudes are also in good agreement.

\section{CONCLUSION}

The $2 \mathrm{~d}$ calculation Mathematica tool only fits $2 \mathrm{~d}$ geometries. If the third dimension is required, the other $3 \mathrm{~d}$ Mathematica tool is used. Current intensities are adjusted afterwards. It has been shown that distributed conductors require intense investigations to produce a non-linear field law in full $3 \mathrm{~d}$ geometry, because high current intensities concentrate the flux in narrow region..

\section{ACKNOWLEDGMENT}

We thank François Méot for proposing the RACCAM project, which allowed us to tackle with this interesting problem.

\section{REFERENCES}

[1] Y. Mori et al., "A 150MeV FFAG synchrotron with "returne-yoke free" magnet", proceedings PAC 2001.

[2] X.-M. Maréchal, "On 2D periodic magnetic fields", ESRF report, SR/ID-90-38.

[3] B. Autin, "Current distribution in a spiral FFAG", private communication (unpublished work).

[4] Y. Ishi, "Accelerator design and construction for FFAG-KUCA ADSR", presentation, Oct. 15th 2004. 